\title{
Evaluation of Endovenous Laser Ablation for Varicose Veins Using a Computer Simulation Model (Secondary publication)
}

\author{
Hisanao Hazama ${ }^{1}$, Masato Yoshimori ${ }^{1}$, Norihiro Honda ${ }^{2}$, Kunio Awazu $1,3,4$ \\ 1: Graduate School of Engineering, Osaka University \\ 2: Institute for Academic Initiatives, Osaka University \\ 3: Graduate School of Frontier Biosciences, Osaka University \\ 4: Global Center for Medical Engineering and Informatics
}

\begin{abstract}
Background and aims: Endovenous laser ablation (EVLA) has been well-reported as a minimally invasive method to deal with varices of the lower extremities. The lasers used fall into two categories: pigment, i.e., hemoglobin-specific lasers in the visible and near-infrared (near-IR) wavebands and longer wavelength mid-infrared lasers where the chromophore is water. The fiber used to deliver the laser energy is also important, and not enough attention has been paid to this element of EVLA. The present study was therefore designed to compare EVLA delivered through two specific fiber types coupled with a near-IR laser wavelength where water was the major chromophore.

Materials and methods: A laser diode system at the wavelength of $1470 \mathrm{~nm}$ was used as the laser energy source near a peak in the water absorption spectrum. Laser energy was delivered with two specific types of optical fiber, a Radial ${ }^{\mathrm{TM}}$ fiber and a Radial 2 ring $^{\mathrm{TM}}$ fiber (CeramOptec, Germany), and EVLA was evaluated using a computer simulation model taking light transport into account based on the Monte Carlo method and temperature distribution with the heat conduction equation. Results and conclusions: It was confirmed from both the simulation model and a previously published ex vivo experiment that carbonization and sticking during EVLA caused by excess temperature rise can be minimized by using the Radial 2ring fiber compared with the Radial fiber, coupled with the $1470 \mathrm{~nm}$ wavelength. In the future, lasers with different wavelengths or optical fibers with differing irradiation modes may appear as candidate systems for EVLA. It is important to evaluate safety and efficacy carefully using the methods in the present study before moving to in vivo indications in human subjects.
\end{abstract}

Keywords: varicose veins • endovenous laser ablation • computer simulation • Monte Carlo method • heat conduction equation

\section{Introduction}

Endovenous laser ablation (EVLA) is a highly effective and minimally invasive treatment for varices of the lower extremities caused by incompetent saphenous veins, where post-operative occlusion rates of the treated veins as high as 95\% have been reported in the literature ${ }^{1)}$. In the clinical setting, near-infrared (near-IR) lasers at wavelengths of $810 \mathrm{~nm}, 940 \mathrm{~nm}, 980 \mathrm{~nm}, 1064$ $\mathrm{nm}, 1320 \mathrm{~nm}, 1470 \mathrm{~nm}$ and $2 \mu \mathrm{m}$ have been used in

Addressee for Correspondence:

Hisanao Hazama

2-1-A14, Yamadaoka, Suita, Osaka 565-0871, Japan

hazama-h@see.eng.osaka-u.ac.jp combination with bare fibers with laser emission from simple flat cleaved end faces. The chromophores of the lasers differ according to the wavelength but can be grossly be grouped into two. Laser energy at wavelengths between $810 \mathrm{~nm}-1064 \mathrm{~nm}$ is absorbed mainly by hemoglobin where coagulation deep into the soft tissue can be achieved without the influence of water. Conversely lasers at wavelengths of $1320 \mathrm{~nm}, 1470 \mathrm{~nm}$ and $2 \mu \mathrm{m}$ are absorbed strongly by water contained in the blood and venous walls. This allows for vasoconstriction at lower energy levels and since the laser energy cannot penetrate deep into soft tissue, a safer

Received date: June 20th, 2017

Accepted date: Nov 11th, 2017 
and more effective treatment can be anticipated. Complications associated with EVLA such as pain of the thighs, bruising, thrombophlebitis, deep vein thrombosis, pulmonary thromboembolism, neural disorders and arterio-venous fistula have previously been reported ${ }^{2)}$ and the usage of lasers with high absorption in water is anticipated to lessen post-operative complications such as pain and bruising. Recent reports state that not only the selection of the wavelength but also the irradiation method affects the treatment outcome and decreases the incidence of postoperative complications such as pain and bruising 3 -7) It is probable that developments and progress of new devices with differing irradiation methods and wavelengths will appear, but in order for such novel devices to be used clinically, they must first obtain regulatory approval through various time consuming and costly non-clinical and clinical trials.

Since clinical outcomes are highly dependent on the experience and technique of the attending surgeon, an objective method for the evaluation of the efficacy and safety of any novel device is warranted. In general, the investigation of the optimal treatment parameters such as wavelengths and irradiation methods is time consuming and costly, but if such investigation can be achieved through computer simulations, it would be highly effective in expediting the process of obtaining the appropriate regulatory approval. In the present study, therefore, the authors analyzed the results of light-heat propagation computer simulation, a combination of calculations of light transport and heat conduction concerning the venous wall temperature changes during an EVLA procedure, based on the premise of using a $1470 \mathrm{~nm}$ laser diode in combination with an ELVeS Radial ${ }^{\mathrm{TM}}$ fiber and an ELVeS Radial 2ring ${ }^{\mathrm{TM}}$ fiber (CeramOptec GmbH, Germany) which can irradiate light laterally. The results of the analysis were compared to that of an ex-vivo experiment using resected veins.

\section{Materials and methods}

In this simulation, calculations were made on the premise that the laser device being used was a regulatory-approved system for EVLA in Japan, a $1470 \mathrm{~nm}$ wavelength laser diode with a maximum output power of $15 \mathrm{~W}$ (LEONARDO ${ }^{\circledR} 1470$ (ELVeS laser 1470 in Japan), CeramOptec). The fibers being used were the ELVeS Radial ${ }^{\mathrm{TM}}$ fiber (not approved in Japan) and the ELVeS Radial 2ring ${ }^{\mathrm{TM}}$ fiber (approved in Japan) both which emit laser energy laterally. Figure 1 shows the positioning of venous tissue, blood and the optical fiber used in the simulation. The assumption was that a blood-filled vein with a lumen diameter of $3.85 \mathrm{~mm}$ was being treated with EVLA using a fiber placed along the vein's central axis. The fiber is retracted at rate of $1 \mathrm{~mm} / \mathrm{s}$ while laser energy is emitted laterally throughout ${ }^{8}$ ). The principle behind the simulation of this light-heat propagation throughout the blood and venous tissue is described below, based on the calculation of light transport by the Monte Carlo model and the calculation of heat transport using the heat conduction equation.

\section{Calculation of light transport}

In order to calculate the light transport through blood and venous tissue three approaches were used: simula-

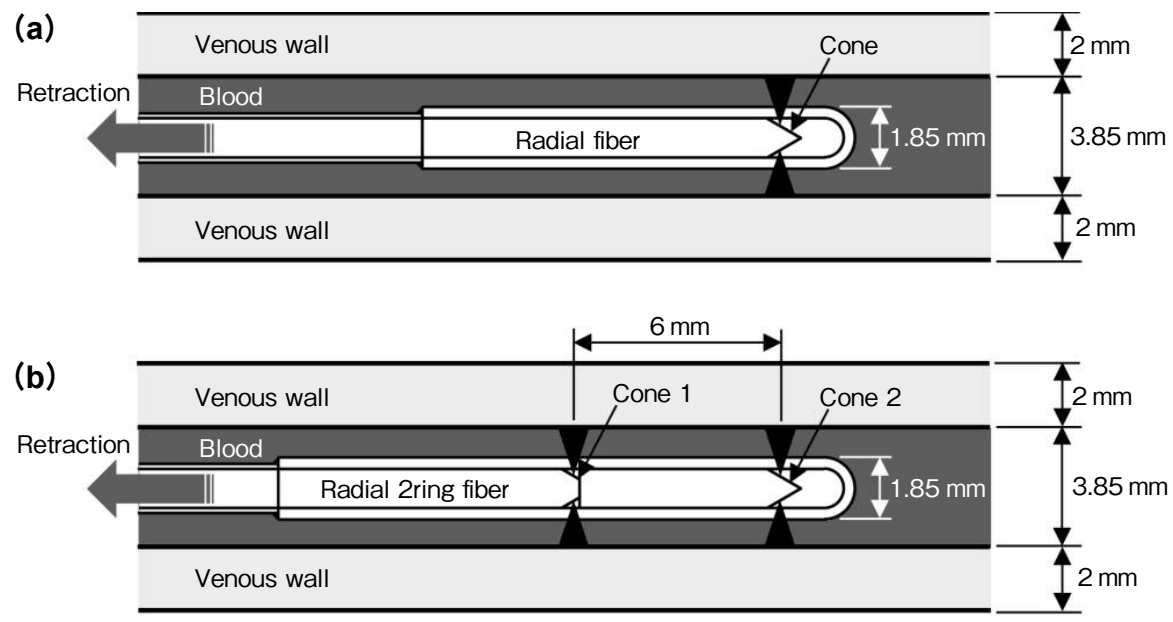

Fig. 1: Schematic of the simulated conditions for EVLA using (a) Radial fiber and (b) Radial 2ring fiber. 
tion codes based on the Monte Carlo method developed by Wang et al.; the Monte Carlo modeling of light transport in multi-layered tissues (MCML); and the convolution for responses to a finite diameter photon beam incident on multi-layered tissues (CONV) 9-11). MCML allows the calculation of light transport within multi-layered structures and in this simulation the layers were set as the blood layer $1 \mathrm{~mm}$ thick from the surface of the optical fiber to the inner surface of the vein, and the venous tissue layer set at a thickness of 2 $\mathrm{mm}$. It was postulated that the blood and venous tissue layers were flat and uniform in structure and light transport was calculated for the diameter or depths ranging to $3 \mathrm{~mm}$. The absorption coefficients and scattering coefficients of blood and venous tissue at the wavelength of $1470 \mathrm{~nm}$, were determined through actual measurements of porcine blood and human venous tissue, as listed in Table $\mathbf{1}^{6,7)}$. The refractive index $1.38^{12)}$ and anisotropy factor 0.9 were taken from the literature ${ }^{13}$ ). For the refractive index of the optical fiber, 1.45 was used, being the refractive index of fused silica at the wavelength of $1500 \mathrm{~nm}^{14)}$. In this simulation, it was assumed that the intensity of the laser energy followed a Gaussian distribution and was incident on blood perpendicularly from the optical fiber. The energy densities of each coordinate of the circular cylindrical coordinate system were calculated, where the laser beam was set as the central axis. Figure 2 shows the laser power distribution of the Radial fiber and Radial 2ring fiber along the central axis measured using a power meter (S122C, Thorlabs) covered by a pinhole with a diameter of $1 \mathrm{~mm}^{6,7)}$. From the results, the beam diameters where the incident laser light intensity is $1 / e^{2}(\sim 13.5 \%)$ of maximum intensity were $1.40 \mathrm{~mm}$ for the Radial fiber and 1.375 $\mathrm{mm}$ and $1.50 \mathrm{~mm}$ for the Radial 2ring fiber as shown as cone 1 and cone 2 in Figure 1b. In this simulation, it was assumed for the Radial fiber that all incident power was delivered laterally in a single cone, whereas for the Radial 2ring fiber the power was delivered from cones 1 and 2 . In the case of the Radial fiber, laser power incident on blood was determined from a ratio between the surface area of a cylinder with a diameter $d$ of $1.85 \mathrm{~mm}$ (the external diameter of the Radial fiber) and a width $w$ of $1.40 \mathrm{~mm}$ and the area of a circle with a diameter same as $w$ of $1.40 \mathrm{~mm}$. On the other hand, in the case of the Radial 2ring fiber, the ratio of the laser power between cones 1 and 2 was assumed to be 16:9 according to the ratio of the respective geometrical cross sectional areas of the

Table 1: Optical properties at the wavelength of $1470 \mathrm{~nm}$ and thermal properties of blood and venous wall used in the simulation model taking account of light-heat propagation.

\begin{tabular}{lccccccc}
\hline & $\begin{array}{c}\text { Absorption } \\
\text { coefficient } \\
\mu_{\mathrm{a}}\left[\mathrm{cm}^{-1}\right]\end{array}$ & $\begin{array}{c}\text { Scattering } \\
\text { coefficient } \\
\mu_{\mathrm{s}}\left[\mathrm{cm}^{-1}\right]\end{array}$ & $\begin{array}{c}\text { Refractive } \\
\text { index }\end{array}$ & $\begin{array}{c}\text { Anisotropy } \\
\text { factor }\end{array}$ & $\begin{array}{c}\text { Density } \rho \\
{\left[\mathrm{kg} / \mathrm{m}^{3}\right]}\end{array}$ & $\begin{array}{c}\text { Specific } \\
\text { heat } C_{\mathrm{p}} \\
{[\mathrm{J} /(\mathrm{kg} \cdot \mathrm{K})]}\end{array}$ & $\begin{array}{c}\text { Thermal } \\
\text { conductivity } \\
\lambda[\mathrm{W} /(\mathrm{m} \cdot \mathrm{K})]\end{array}$ \\
\hline Blood & 18.3 & 176 & 1.38 & 0.9 & 1048 & 3598 & 0.552 \\
\hline Venous wall & 18.2 & 151 & 1.38 & 0.9 & 1020 & 3450 & 0.501 \\
\hline
\end{tabular}
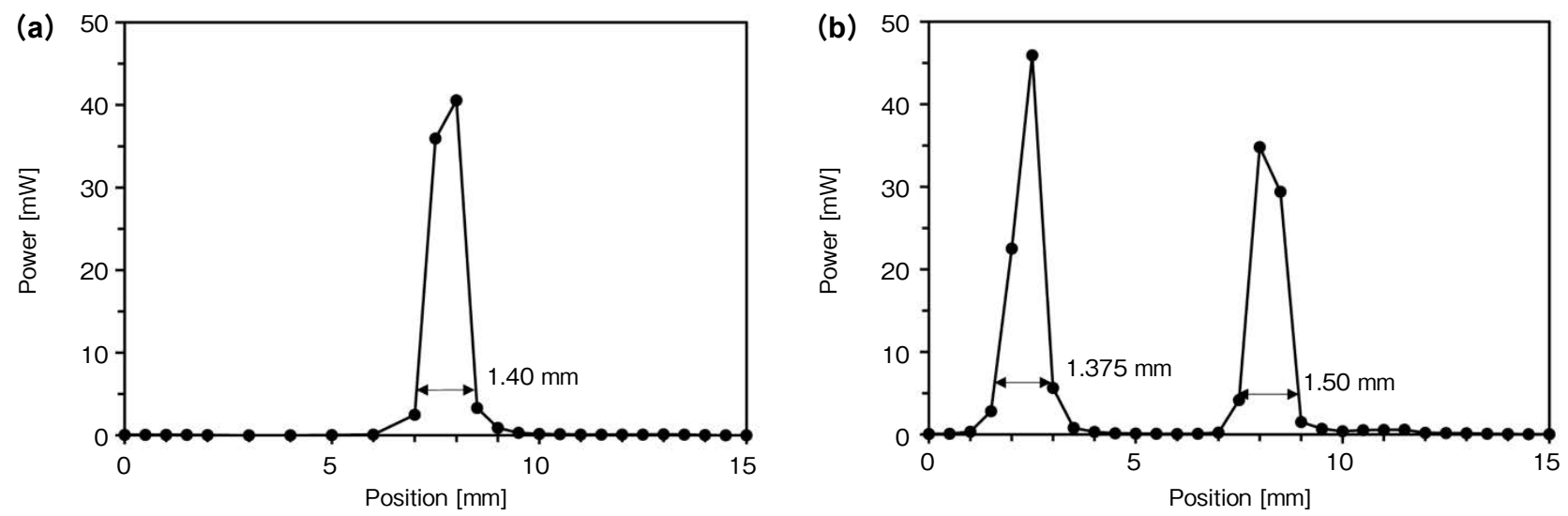

Fig. 2: Laser power distribution at the surface of the (a) Radial fiber and (b) Radial 2ring fiber along the central axis measured using a power sensor covered by a pinhole with a diameter of $1 \mathrm{~mm}$. 


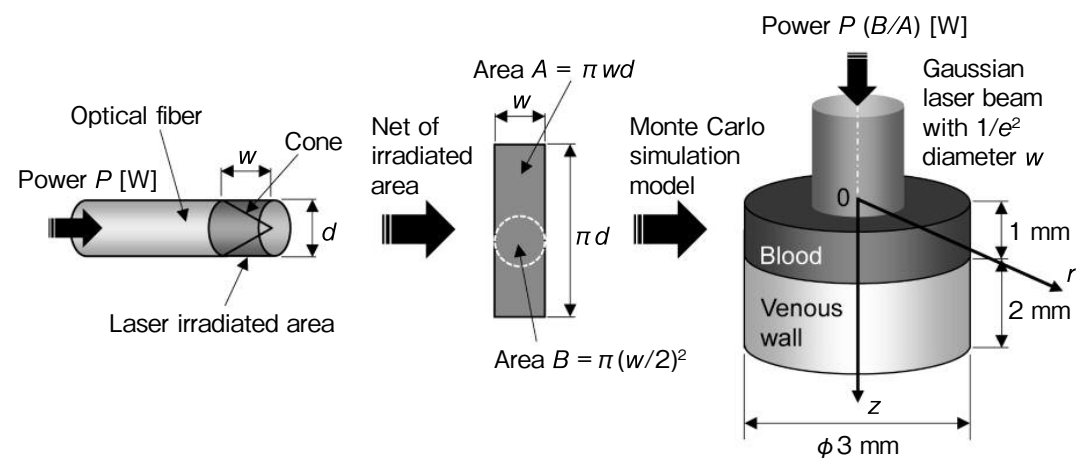

Fig .3: Schematic of the calculation of the laser power used as the input for the computer simulation and the model used in the Monte Carlo simulation.

cones. Since the external diameter of the Radial 2ring fiber is $1.85 \mathrm{~mm}$, laser power incident on blood for the cone 1 or cone 2 was determined from a ratio between the surface area of a cylinder with a diameter $d$ of 1.85 $\mathrm{mm}$ and a width $w$ of 1.375 or $1.50 \mathrm{~mm}$ and the area of a circle with a diameter same as $w$ of 1.375 or 1.50 $\mathrm{mm}$, respectively. Although in actual use both the Radial and Radial 2ring fibers emit laser energy forwards $4,6,7)$, in this simulation this was disregarded in order to simplify the simulation. The number of MonteCarlo simulation trials (number of incident photons) was set at $10^{6}$ and the grid size for the calculation was set at $50 \mu \mathrm{m}$ for both depth and radius.

\section{Heat transport calculation}

From the intra-tissue energy density distribution attained by the Monte-Carlo simulation, temperature rises and their temporal changes due to heat conduction were calculated through numerical analysis. Whereas a cylindrical coordinate system with an axis along the center of the laser beam was used for the energy density distribution calculated with the light transport simulation, the 2-dimensional heat conduction equation shown below was used for the calculations of heat transport in order to simplify the calculations. The energy density attained from the MonteCarlo simulation was substituted for the energy density of the heat source $S\left(\mathrm{~W} / \mathrm{m}^{3}\right)$ of the heat conduction equation and the thermodynamic temperature $T(\mathrm{~K})$ was calculated for any given time $t$ when the heat source moved in the same manner as the retraction of the fiber shown in Figure 4.

$$
\rho C_{\mathrm{p}} \frac{\partial T}{\partial t}=\lambda\left(\frac{\partial^{2} T}{\partial x^{2}}+\frac{\partial^{2} T}{\partial y^{2}}\right)+S
$$

The density $\rho$, specific heat $C_{\mathrm{p}}$ and thermal conductivity $\lambda$ used in this calculation are listed in Table $\mathbf{1}$ and are those cited in the literature for blood ${ }^{15-17)}$ and for the venous wall ${ }^{18)}$. The above equation was dis- cretized using a finite differential method, i.e., the Crank-Nicolson method by employing the central difference along the time axis. The obtained solution was converged using the successive over-relaxation method. The temporal profiles of the temperatures of each coordinate were calculated at a time step of $0.1 \mathrm{~s}$. The initial temperature of the blood, vein and fiber was set at $37^{\circ} \mathrm{C}$, and the boundary condition for the bilateral and lower boundaries was fixed at $37^{\circ} \mathrm{C}$. Additionally, the temperature of blood in contact with the optical fiber $(y=0 \mathrm{~mm}$ in Figure 4) was used as

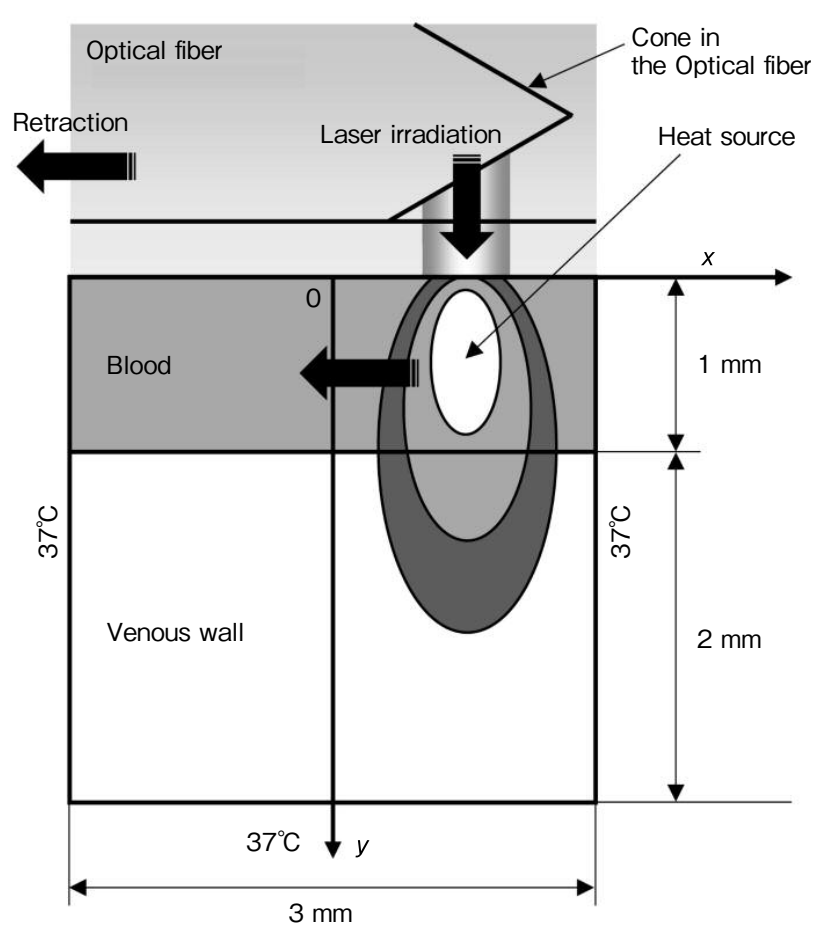

Fig. 4: Schematic of the computer simulation model to estimate the temperature distribution by solving the heat transfer equation. 
the fiber temperature at $0.1 \mathrm{~s}$ later. In this simulation, in order to compare the Radial fiber and Radial 2ring fiber in the simplest of settings, complex phenomena such as bubble formation, morphological changes in venous tissue, and histologic changes accompanying coagulation were not taken into consideration.

\section{Results and discussion}

The results of the simulation, temporal profiles of the temperature at the vicinity of the surface of the venous wall, i.e., at the position $x=0 \mathrm{~mm}, y=1.05$ $\mathrm{mm}$ in Figure $\mathbf{4}$ at laser powers of 5.8, 8.9, and 11.8 $\mathrm{W}$ and a fiber retraction speed of $1 \mathrm{~mm} / \mathrm{s}$ are shown in Figure 5. It is clear that the maximum temperature of the vein wall is lower when the Radial 2ring fiber was used at all laser power settings. The purpose of EVLA is to constrict and obstruct the vein through coagulative denaturation. In order to prevent unnecessary carbonization and vaporization, it is thought that a temperature less than $100^{\circ} \mathrm{C}$ should be maintained for a certain period of time. From the results shown in Figure 5, the temperature grossly exceeded $100^{\circ} \mathrm{C}$ when the Radial fiber was used at laser powers of 8.9 and $11.8 \mathrm{~W}$ which means that excessive heating for the purpose of EVLA has occurred. On the other hand, when the Radial 2ring fiber was used, the maximum temperature at the laser power of $11.8 \mathrm{~W}$ was $85^{\circ} \mathrm{C}$ lower than that of the Radial fiber, hence there was less risk of carbonization and vaporization compared to the Radial fiber at the same laser power.

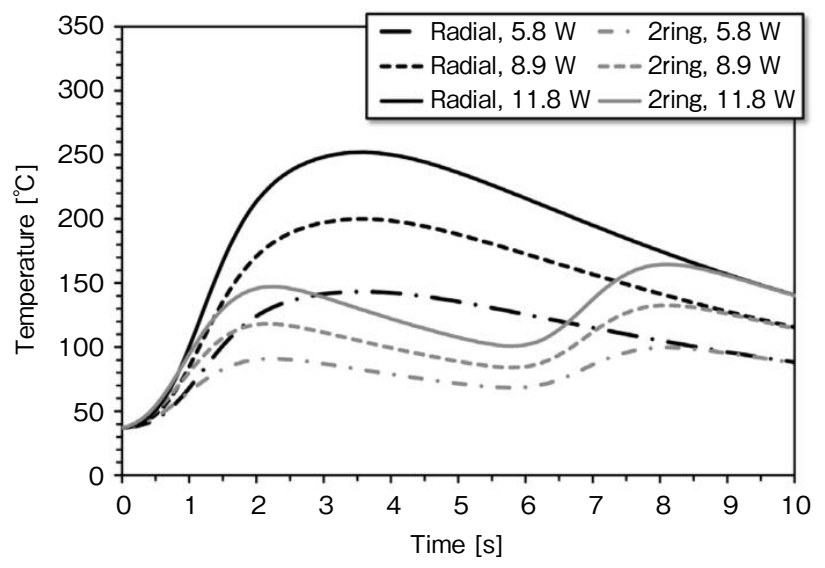

Fig. 5: Temporal profiles of the temperature at the vicinity of the surface of the venous wall, i.e., at the position $\mathrm{x}=0 \mathrm{~mm}, \mathrm{y}=1.05 \mathrm{~mm}$ in Fig.4.
In a study in which ex vivo experiments were actually performed under the same conditions as the present simulation, namely a wavelength of $1470 \mathrm{~nm}$ and a fiber retraction speed of $1 \mathrm{~mm} / \mathrm{s}$ using porcine blood and human venous tissue, stated that when a Radial fiber was used, successful vein constriction with no carbonization or perforation of tissue was achieved at a laser power of $5.8 \mathrm{~W}$, however at laser powers of 8.9 and $11.8 \mathrm{~W}$ sticking of the fiber was seen in 40 and $60 \%$ of the attempts, respectively ${ }^{7)}$. In this same study successful vein constriction without carbonization, perforation of tissue and sticking of the fiber was achieved at all laser powers when the Radial 2ring fiber was used.

The present study has some limitations. In the present simulation, the aspect of forward laser emission from the fibers was ignored and the model was based on the assumption that blood and the venous wall would present a smooth plane. Also complex phenomena such as bubble formation, venous tissue distortion and histological changes accompanying coagulation were not taken into consideration. Due to the omission of such considerations, the absolute values of the calculated temperatures contain inherent errors which could be large. The induction of latent heat accompanying the phase change of blood from liquid to vapor was not taken into consideration, and this may have led to an overestimation of temperature in the simulation, but the tendency seen in the simulation sufficed to explain the results of the previously reported ex vivo experiment carried out under the same conditions as the present simulation, and with the same types of optical fiber. Improving the precision of the simulation according to necessity may further help when designing new laser fibers and laser light sources, and planning EVLA treatments in the a clinical setting.

The reduction of costs and time involved in the regulatory approval of new medical devices to be used in the clinical setting is very important. Simulations can effectively search for optimum laser irradiation parameters through exhaustive trials and evaluations which is very difficult to do in a clinical trial, and also produce highly reproducible, quantitative evaluations under even the most extreme of conditions in a short period of time. Even further, simulations can be used for the theoretical elucidation of clinically occurring phenomena such as in this EVLA simulation, and lead to furthering the safe and effective clinical applications of EVLA. 


\section{Conclusions}

The present study as an established theoretical simulation confirmed that the combination of a $1470 \mathrm{~nm}$ laser diode with a Radial 2ring fiber used for endovenous laser ablation resulted in less excessive heating of venous tissue and avoided carbonization and sticking compared to that produced by the same laser used with a Radial fiber. This confirmation is made through both our computer simulations, and the results of an ex vivo study performed under exactly the same conditions as the simulation. In the future, lasers with different wavelengths or optical fibers with differing beam delivery modes may appear. In order to evaluate the novel devices for clinical application, the light-heat propagation simulation using the Monte-Carlo simula-

\section{References}

1: R. van den Bos, L. Arends, M. Kockaert, et al.: Endovenous therapies of lower extremity varicosities: A meta-analysis. The Journal of Vascular Surgery, 49: 230-239, 2009.

2: M. Hirokawa: Current status of endovascular treatment for varicose veins. Journal of Japanese College of Angiology (in Japanese), 49: 239-245, 2009.

3: L.S. Kabnick, J.A. Caruso: EVL ablation using jacket-tip laser fibers: Treatment outcomes reveal improved postoperative recovery in endovenous laser ablation patients. Endovascular Today, 77-81, July 2009.

4: R. Sroka, K. Weick, M. Sadeghi-Azandaryani, et al.: Endovenous laser therapy - application studies and latest investigations. Journal of Biophotonics, 3: 269-276, 2010.

5: S. Nozoe, N. Honda, K. Ishii, et al.: Comparison of the efficacy and the safety in endovenous laser ablation at wavelengths of $980 \mathrm{~nm}$ and $1470 \mathrm{~nm}$ laser based on human vein tissue optical properties. The Journal of Japan Society for Lasers in Surgery and Medicine (in Japanese), 33: 7-14, 2012.

6: S. Nozoe, N. Honda, K. Ishii, et al.: Ex vivo investigation of optimal irradiation parameters in endovenous laser ablation at wavelengths of $980 \mathrm{~nm}$ and $1470 \mathrm{~nm}$, The Journal of Japan Society for Lasers in Surgery and Medicine (in Japanese), 34: 372-381, 2014.

7: S. Nozoe: Ex vivo investigation of optimal irradiation parameters in endovenous laser ablation. Master's thesis, Division of Sustainable Energy and tion of light transport combined with solving of the heat conduction equation, as used in the present study, proved extremely effective. Utilizing computer simulations may not only enhance the efficiency of novel equipment designs but also may help in structuring theoretical interpretations of results gained through clinical or non-clinical trials. Highly reproducible and quantitative evaluation of laser irradiation parameters can be performed in short periods and therefore computer simulations may become extremely valuable for promoting clinical application of many novel medical devices, and not just limited to those used for endovenous laser ablation.

Disclosure of conflicts of interests No conflicts of interests exist

Environmental Engineering, Graduate School of Engineering, Osaka University (in Japanese), 2013.

8: R. Blagova, C. Burgmeier, S. Steckmeier, B. Steckmeier, et al.: Ex-vivo investigations on endoluminal laser therapy of varicosis - An optimization process. Medical Laser Application, 22: 242-247, 2007.

9: Monte Carlo Simulation Package, Webpage of Optical Imaging Laboratory at Washington University in St. Louis, http://oilab.seas.wustl.edu/mc.html.

10: L. Wang, S.L. Jacques, L. Zheng: MCML - Monte Carlo modeling of light transport in multi-layered tissues. Computer Methods and Programs in Biomedicine, 47: 131-146, 1995.

11: L. Wang, S.L. Jacques, L. Zheng: CONV - convolution for responses to a finite diameter photon beam incident on multi-layered tissues. Computer Methods and Programs in Biomedicine, 54: 141150, 1997.

12: V.V. Tuchin: 1.6.2 Refractive-index variations of tissue, Tissue Optics: Light Scattering Methods and Instruments for Medical Diagnosis (2nd Edition), 104-106, 2007, SPIE Press.

13: V.V. Tuchin: 1.8.2 Concepts of phantom construction, Tissue Optics: Light Scattering Methods and Instruments for Medical Diagnosis (2nd Edition), 132-137, 2007, SPIE Press.

14: SIGMAKOKI Co. Ltd.: Opto Sigma General Catalog 01 First Edit. (in Japanese), B329, 2014.

15: T. Kenner: The measurement of blood density and its meaning. Basic Research in Cardiology, 84: 111124, 1989. 
16: M. Mendlowitz: The specific heat of human blood. Science, 107: 97-98, 1948.

17: K. Tanishita, Y. Nagasaka, A. Nagashima, et al.: Measurement of thermal conductivity of blood by transient hot wire method. Transactions of the
Japan Society of Mechanical Engineers Series B (in Japanese), 47: 1784-1791, 1981.

18: B. Choi, A.J. Welch: Analysis of thermal relaxation during laser irradiation of tissue. Lasers in Surgery and in Medicine, 29: 351-359, 2001.

\section{[Acknowledgements]}

Editor's Note: This paper was originally published in Japanese in The Journal of Japan Society for Laser Surgery and Medicine, Vol.37-2: 175-180, 2016, and has been specially translated for inclusion in Laser Therapy as an English Original Article. 